This is the accepted manuscript version of a chapter from Singh G (2019) The Death of Web 2.0: Ethics, Connectivity and Recognition in the Twenty-First Century. Abingdon: Routledge

\title{
Chapter Seven Social Media as a False-Self System
}

In the Charlie Brooker-helmed television drama Black Mirror (Ch4/Netflix 2011-present), there is a moment in the episode 'The Entire History of You' when the two main characters Liam and Ffion (played by Toby Kebbell and Jodie Whittaker) are having sex in their marital bed. We are witness to their lovemaking as a discernibly passionate encounter full of physical satisfaction, signified through energetic heavy-breathing, moaning and sweat. We see the couple enthusiastically snatch at each other's bodies, apprehending each other in a grasp of mutual body-recognition - one in the other, each as one whole part of a greater union. It is, in other words, a representation of an idealised sexual encounter between two people who would seem very much in love. The scene is abruptly intercut with another in which the same couple, in the same bed, are still having sex - but something altogether disturbing is revealed in this alternating effect. The premise behind 'The Entire History of You' is based around speculative prosthetic memory technologies, where nearly all of the characters in the episode have had a small device implanted in their head called 'grains'. These implants record everything they see, and from these recordings, they are able to rewatch (and thus to an extent, relive) moments from the past, either privately, or in company through television monitoring. This documentation lifestyle is very much an extrapolation of the ways in which people have adopted social media technologies to share abundant images of their lives. From holiday snaps to breakfast to ultrasound images, photo albums and social media galleries and 'stories' act as evidence to satisfy the lifestyle clarion call of 'pics or it never happened'. It is also reflective of the normalisation of this evidential behaviour characters who have not had 'grains' fitted are considered curiosities, anomalies: much like those who have not adopted Facebook and other massively popular Web 2.0 technologies in real life, they are outliers.

Throughout the episode, various characters use the playback function to re-examine aspects of their lives. Most instances are fun and rather trivial, involving selfies and the production of other recordings that can be watched in company on a television screen. In the scene described above, it is revealed to us through intercutting that Liam and Ffion use the 
memory technology to watch playback from recordings of their lovemaking past, in order to augment the sex of the present; each viewing their own perspective privately. The revelation for us as viewers, sutured into a complicity with the characters thanks to the extensive use of point-of-view shots in the episode, is the disturbing realisation that both of the characters are entirely focused on their own playback image, rather than the person they are actually having sex with. This masturbatory mode of technologically-enhanced sexual activity is made all the more alienating to watch because of the blank expressions on Liam and Ffion's faces - exacerbated by the way the technology makes their eyes turn pure white when viewing private playback. The sex is consensual, there is little doubt on that count - and a conciliatory kiss is shared by the couple once the sex is finished. However, one is left with the feeling that both characters are deeply into a privatised experience of sexuality - transactional, almost. The kiss signifies care, but there is a discernible distinction made between that, and the sexual act, during the course of this particular scene.

The main problem, somewhat ironically (given that many popular cultural representations of autosexual activity focuses on the relative dysfunctionality of masturbation), relates to the fact that neither character is alone - they are actually in the physical presence of another, and so to borrow from Sherry Turkle's phrase once more, they are 'alone together' (2011). In some ways the facilitation of solitary fantasy might be more understandable given that the other would not be present. In fact, one of the other characters relays such a use during a dinner party scene. Furthermore, one might imagine this technology in a more positive light where, say, a partner is away working and physical intimacy is simply impossible; or perhaps where a disability might hamper physical mobility, and such technology is used as an augmentation. In such cases, one might speculate that technology can facilitate a relationship. But the scene described in Black Mirror is a case of an altogether different kind: it looks routine, perfunctory, joyless. On the one hand, the characters have literally objectified one another's bodies, for the sake of reliving a past sexual encounter, and then adding a real-time sexual component to it; on the other, they have both literalised the objectification of the other as an image, rather than as a whole person. Indeed, the impression we are given is that the other body in this situation could be anyone's. However, because they happen to be married to the person they are watching, and to the person they are having intercourse with (although the two experiences are on 
distinct experiential registers), no adultery is being committed. The evidence from this scene, may be summed up in that to an extent, both parties in the marriage are on fairly safe moral ground, but ethically-speaking, there are issues around the disruption of mutuality in a fully-matured relationship that are troublesome. In addition, the moral status of Ffion's actions come under scrutiny later in the episode, as do the ethics of Liam's actions in the use of the technology and the consequences he sets in motion as a result.

Of course, such disturbing imagery is easy enough to condemn, but the realities of married life, with children in the mix, are challenging, and a technology that could conveniently augment one's marital expectations might be viewed as a straightforwardly transactional solution. Indeed, these are issues that form a favourite subject of discussion in both popular culture, and on the psychotherapist's couch. In The Divided Self (1965: 86), R. D. Laing describes a case in which one of his patients, who otherwise conducted his life along normal lines:

[...] could never have intercourse with his wife but only with his own image of her. That is, his body had physical relations with her body, but his mental self, while this was going on, could only look on what his body was doing and/or imagine himself having intercourse with his wife as an object of his imagination.

This account describes in quite stark terms, the fullest expression of false-self as a normal pathology in Laing's thought, and reflects, psychologically, what is literalised in 'The Entire History of You'. Not necessarily an aspect of contemporary culture that is unfamiliar - we do, after all, live in image-driven times - nonetheless, Laing drives home the objectification apparent in everyday life which has the power to disrupt congress, impoverish what we are told should be a moment of absolute intimacy with someone we love the most, through an existential pattern of objectification and alienation. The relative pleasures which one may experience as a sexual and sexualised being are here offset by a psychic deadness, and a disconnect between the inner world of one's emotions and the outer world of social being. It is problematic enough, one suspects, to even countenance the discretion with which inner and outer are treated here - as a binary, as if the two are somehow separate aspects of consciousness. But given that Laing's work is often mobilised through dialectical-, rather 
than picture-thinking, once suspects that this is not his emphasis. Rather, the binary is a symptom of the disconnect, and that this disconnect forms through the breakdown of mutuality as a principle in our most valued relationships.

So far in Part II of this book, I have discussed similar descriptions of recognition (and its disruption) from a number of fields relevant to the study of interpersonal communications in Web 2.0 contexts. Having outlined Honneth's rich description of recognition in light of the notion of 'reciprocated confidences' and the 'reciprocally-constitutive', I offered a strategy whereby Honneth's application of object-relations theory to the concept of recognition could be reinforced through the self-psychology of Heinz Kohut, and in particular Kohut's pathology of narcissistic success and failure. This psychosocial mechanism of narcissism in self-psychology is the same mechanism that affords the development of mutuality through confidence, respect and esteem, in social interaction, and ultimately provides the foundation to enable human actors to develop empathy. I progressed this approach by describing Kohut's transhistoric narrative of self-realisation, as a narrative that embodies an interpersonal principle of mutuality that encapsulates recognition as a bodily-emotional, moral and social phenomenon; and it is this aspect of self-realisation that allowed me to tentatively outline a depth and relational approach towards ethical personality (in Honneth's parlance) in social (media) relations.

In this final chapter, I wish to elaborate the depth dimension of this approach, by expanding on my discussion of self-realisation as a psychosocial phenomenon that hinges upon the recognitive principle of mutuality. I shall do this through discussion of classical and postJungian thinking on persona, as well as drawing from other psychological schools. I then engage a specific derivation of the term 'complex' from Jungian depth psychology to explore the intricacies of authenticity as a recognitive notion in social media interactions. The complex in this sense brings together the building blocks of the psyche, often experienced as partial personalities in the Jungian terminology, but in addition raises the question of 'cultural complexes' as constellations of psychic and social space interacting and mutually impacting. This is, specifically, a psychosocial development of the original term in postJungian thought and psychotherapy (Singer 2000; Singer and Kimbles 2004; Singer 2004; Kimbles 2000). In what follows in this current chapter, I hope to make an original 
contribution to the understanding of the psychology of online interaction, within a framework that might describe social media as a false-self system. I begin my discussion by developing my application of Laing and his existential approach to psychology. In considering his contribution to our understanding of separateness and relatedness as an existential equilibrium, we might begin to further understand this as underpinning broader social interaction in the relationship between self and other, and how Web 2.0 technologies amplify this equilibrium.

\section{Personae, Separateness and Relatedness}

The existential-phenomenological work of the psychiatrist and psychotherapist, R. D. Laing has been influential in my thinking on the development of personality, social interaction, and in particular, familial relationships and their role in the development of an 'authentic' sense of self. I fully acknowledge that Laing's approach, and some of his practices, were not without controversy. However, his overview of the existential equilibrium of separateness and relatedness in everyday pathology, as developed in his influential book, The Divided Self (1965) ought to be of particular interest to students of social media. The somewhat underacknowledged observations he made on the notion of authenticity in one's attitude to the self, and the parasocial interface of identity, have immediate relevance for the ways in which contemporary connectivity shapes how we see ourselves, in relation to others. It underpins a sense of self-recognition (through aspects of what might be framed in popular discourse as self-esteem) and also the recognition of others (especially in the sense of otherregarding, and empathy). When read retrospectively, in some ways to my mind at least, Laing's work also pertains to a distinctive Jungian tone. This is especially discernible in the implications of his acknowledgement of the role of the imago, the psychological Image, so to speak, in the relationship between self and other. His approach draws explicitly from the Jungian notion of persona as a structuring archetype. For example, he writes (1965: 95) that

'A man without a mask' is indeed very rare. One even doubts the possibility of such a man. Everyone in some measure wears a mask, and there are many things we do not put ourselves into fully. In 'ordinary' life it seems hardly possible for it to be otherwise. 
This concept of social masks, worn to suit the occasion, is a familiar one; as is its application to social media interactions, and to Web entrepreneurship, where microcelebrity depends largely upon reaching audience segments in ways that tap into popular typology. This is a conscious strategy, to build alignments of identification or sympathy, and ultimately, audience engagement (and emotional, parasocial investment).

The etymology here is instrumental. As I have often discussed with students when examining the performative aspects of Instagramming, or Facebook status updates for example, the etymology of persona works as a reminder of what we might already intuitively know about persona as a psychosocial mechanism. The persona in ancient Greek theatre, referred to the mask a performer wore, symbolising a character's traits and representing something of the character's inner nature or essence. It signified the true role of the character in the drama, for the audience to evaluate and engage with. The performance, through use of the persona, invited the audience to infer qualities inherent in the wearer, encouraging a belief in the verisimilitude of performance, where the masquerade is fully acknowledged as a representation. The performative or dramaturgical aspects of this have not gone unnoticed social media scholarship, where aspects of performativity lend themselves to the notion of masquerade, and aspects of dramaturgy lend themselves to notions of staging, iconography and mise-en-scene. These kinds of approaches are noted particularly in social interactionist approaches where the application of Goffman's notion of performance of self in everyday life drew heavily from the accoutrements of theatre, staging and performance as an analogy for self-presentation (Athique 2013; Goffman 1997; Papacharissi 2010; Walker Rettberg 2008, 2014).

One may take this analogy further, by considering the allegorical and psychic aspects of selfpresentation in social interactions: Jung used the notion of persona to describe the phenomenon of social role-playing as a structuring psychic phenomena (or archetype), complementary to the social mechanism employed in interpersonal communications. To put it another way, persona in the Jungian sense, is the psychic structuring presence refracted through the social face an individual presents to the world. It is a psychological figure that speaks to both inner and outer dimensions of one's consciousness, and is essential to selfexpression in social situations, to the relative stability of personality, and to the 
establishment and maintenance of mature relationships. In his Two Essays on Analytical Psychology (1953), Jung described the archetypal notion of persona as '[...] a kind of mask, designed on the one hand to make a definite impression upon others, and on the other to conceal the true nature of the individual' (1953: 190). So, for Jung (pre-empting Laing), persona performs this dual masking function of revelation and concealment to protect the sense one has of a coherent self. This facilitates the interpersonal aspects of social life by enabling individuals to enter into the risky business of social relationships, through both opening 'oneself' to others and through protecting those aspects of the personality that lie in a more fragile state, akin to ego. This is similar in many ways to the 'tension gradient' between the various constituents of self, found in Kohut's self-psychology as discussed in Chapter Five, where the materials of our social interactions are defined and realised through the distinctions and continuities of self- and other-regarding. This is done, ultimately, in a trust-themed everyday drama where both conscious choices and unconscious notions of what we ultimately share of ourselves with others, define the relationships we find ourselves in.

In his own approach to this relationship structure, Jung tried to account for the depth and complexity of the human personality, in its dialogical nature. This dual function of revelation and concealment is similar in many ways to subsequent theorisation of the psychological notion of 'false-self': a social face of personality and that aspect of oneself that presents in interpersonal interaction. We have already seen similar notions in the example of Laing, but this notion additionally appears in several different traditions in psychology. In another wellknown example, Donald Winnicott wrote that the false-self is a defensive mechanism, designed to facilitate personal relationships through 'compliance' with what others might expect of us (1965). As with Laing's approach, and to an extent with Kohut, there is a sense in Winnicott's version of false-self, especially in more extreme pathological cases, that the presentation of self is a show, masking a feeling of inner deadness or a creeping sense of inauthenticity.

In this version, the false-self works in social contexts by deflecting from others' suspicion of one's detachment from relationships and the world. It also reflects Jung's version in which there is an everyday normal pathology of feeling fake or phoney in some way that leads the 
individual ego to build a deception or persona as a defence mechanism against emotional distress. In Winnicott's approach, this is built up in infancy, and is subsequently reinforced through life, as relationships develop or fall away, and as our social networks are established. Through unconscious processes of introjection, internalisation of the experience of others and, in more extreme cases, through the imitation and internalisation of other people's behaviour, this false-self comes to be (mis)recognised by others and even by oneself as the whole thing, the 'real me'. This has real-world implications for which accelerated forms of misrecognition have developed in social media interactions. I have written about the specifics of these implications elsewhere (2017), and this was the topic of discussion in Chapter Four, referring to misrecognition as social disrespect.

Although the same individual could 'put on' different personas (or personae) in order to deal with the various relationships in his/her life (e.g. wife, mother, sister, daughter, employee, carer, party animal), these are not just conscious decisions and personal choices made to suit the social occasion. For Jung, in the proper sense, the persona is part of an intrapsychic apparatus, an ego operation interfacing between one's internal experiences and one's social being. Where there are obvious, conscious choices to be made in social interactions, the psyche uses the less-conscious operations of persona to cope with the demands of maintaining sophisticated social relationships by mediating between the internal world and the external world. Notions of acceptance and acceptable behaviour, awareness of context, collaboration and competition - these interactions all demand a complex social interface such as persona, because they impact upon the integrity of one's personality and one's status as a person with distinct, autonomous interests. To put it another way, social compliance places a number of demands upon the individual to curb unfettered self-ish-ness, and a functioning persona copes with these demands, helping to facilitate a coherent and continuous sense of self. Although rarely referring explicitly to theories from classical Jung (a notable exception being Balick 2014b), and even more rarely relating to disclosure practices that amount to much beyond conscious strategies for finessing social management, these aspects of social interface are fully acknowledged in some approaches to the critical study of social media. For example, Mendelson and Papacharissi (2011: 252) note that 
In everyday life, people consciously and unconsciously work to define the way they are perceived, hoping to engender positive impressions of themselves. This effort entails emphasizing certain characteristics, through dress, hairstyle, behavior and/or speech, while hiding or diminishing other characteristics perceived as flawed, depending on the context.

This is literalised in everyday practices of 'Impression Management' in social media and other online interactions. In fact, it is commonplace to use different social media applications to facilitate different kinds of social interactions, expectations, and levels of relationship intimacy. A number of studies, including social research of practices in interactions between teenagers (e.g. Vandebosch et al 2013) show that it is common practice to manage multiple FB and SNS profiles for different audiences, expressing different public faces or personae for different situations. For example, so-called Rinsta and Finsta profiles - respectively, a 'real Instagram' profile to interact with one's peers, and a 'fake Instagram' profile to work as a decoy to assuage parental anxiety around 'stranger danger', grooming and other perceived threats to children. The Finsta account is the one that (typically) a teenager will allow a parent full access to, using it as a front of sorts to distract from activity disclosed on the real account that might be deemed by an authority figure as inappropriate, shameful or prohibited.

It ought to be noted that although this is ostensibly deceptive behaviour that most parents would hesitate to endorse, it is one mechanism among many through which teenagers are able to explore and develop their sense of identity independent of an authority whose psychic image comes to symbolise restriction and curtailment upon the development of a dignified self with autonomous interests. In Jungian terms, this commonplace adolescent response may be read as the 'trick' to counter the parental trickster, whose operations materialise through surveillance technologies and practices of supervision (as discussed by Samuels, 1993). Such are the psycho-political tensions pulling at the heart of the separateness-relatedness dialogue in contemporary adolescent life.

On these questions one might return to Laing for a moment. We may also think of his model of existential equilibrium at the heart of separateness-relatedness as a departure from the 
kinds of self-certifying subjectivity found within many classical strands of psychoanalysis. This is thanks in part to his acknowledgement of the phenomenological aspects of interconnectedness between mind, body and world. This is an influence from French existentialism and in particular, the work of Maurice Merleau-Ponty. Merleau-Ponty openly saw mass media forms of the Twentieth Century, the cinema especially, as materially representative of this interconnectedness (1964). Perhaps most of all, aside from his development of the existential approach to imaginative misrecognition of an authentic self in relation (especially to others - the focus of discussion in previous chapters, and a topic I shall reprise in the next section on data identity) we find in Laing a significant departure from the kinds of recognition and authenticated difference found in, for example, the Lacanian infant. It is enough, for Laing (and echoing my comments regarding the Jungian 'trick' some moments ago), that we are able to acknowledge the capacity to experience oneself as autonomous: 'that one has really come to realize that one is a separate person from everyone else. No matter how deeply committed in joy or suffering to someone else, he is not me, and I am not him' (1965: 52).

This has perhaps obvious applications in the way that we might think of the role of social media interactions in the development of adolescent identity more generally, and in the social and cultural practices of adolescents whose lives so often embrace the accelerated convenience of social media applications. Additionally, in this realisation one might also acknowledge the important fact here that late capital societies in general have committed to the status of autonomous subject a role as some sort of barometer of free will. That is to say, individuation-as-separateness is prized to the degree that, on a psychosocial level and within a psychic economy, there appears to have been a fall in the currency of the 'relatedness' vector in the existential equilibrium.

Separateness seems to be considered more valuable, politically- and economically-speaking. Aside from institutional commitments such as to one's family (as an institution especially), to community (in the institutional arrangements of such, like taxation, charity donations, volunteering and so on) and democracy (a commitment to the procedures of the State, as well as to consumerism) relatedness disappears from view. This general shift is noticeable in the kinds of loneliness and lack of solitude as discussed in Chapter Five (see also Turkle 
2015), typical of social interactions in contemporary accelerated media ecosystems characterised by always-on, persistent connectivity. It is also reminiscent of the way that the experience of capital has been narrativised in the historiography of Western democracies more generally, as outlined by Frederic Jameson $(1981,1988)$ and Max Weber before him (2001). This kind of existential disequilibrium therefore sits within the logic of late capital, as the privatisation of experience intensifies, and one can imagine the gradual dissipation of mutuality accelerate in pace through the myth of connectivity.

The lack of reflective and recognitive space necessary to foster empathy, as I have already outlined in previous chapters in relation to Sherry Turkle's work in particular, may be indicative of this problem. However, Laing goes further than this in relation to the feeling of autonomy. If we are compelled in this upwards movement, towards individuation (in the late capital, rather than the Jungian, sense of the term), then we are robbed of the fullest experience of autonomy such that for Laing, one 'can experience neither his separateness from, nor his relatedness to, the other in the usual way' (1965: 52). The existential equilibrium between a sense of separateness and a sense of relatedness is crucial, for Laing, to enable individuals to cope with the reality of existential disquiet as social beings. This existential disquiet is the lived knowledge that, expressed in Sartre's terms, one cannot love for someone else, nor can they do the same for me (2001); or in Heidegger's thought that we cannot die another's death, only our own, towards which, we are thrown (2000).

\section{The Benign and Vicious Circles of Data Materialisation}

The added complication of living the disequilibrium through which we hurtle towards a mythic point of self-actualization or individuation is that a condition of persistent, semipermanent connectivity requires that we give something up willingly in order to participate in this journey. It is worth quoting Laing in full here (1965: 52-53), to illustrate the 'something' we give up:

A lack of sense of autonomy implies that one feels one's being to be bound up in the other, or that the other is bound up in oneself, in a sense that transgresses the actual possibilities within the structure of human relatedness. It means that a feeling that 
one is in a position of ontological dependency on the other (i.e. dependent on the other for one's very being), is substituted for a sense of relatedness and attachment to him based on genuine mutuality. [...] Therefore, the polarity is between complete isolation or complete merging of identity rather than between separateness and relatedness. The individual oscillates perpetually between the two extremes, each equally unfeasible.

This oscillation is compounded in the contemporary media landscape by the fact that the entire ecosystem is underpinned by (and is reliant upon) networks of data. Several recent studies that engage with aspects of political economic critique of data generation, management and monetisation (e.g. Allmer 2015; Fuchs 2012, 2013, 2014; McChesney 2013; Srnicek 2017; Terranova 2004) tend to highlight the enormity and sheer amount of data, and the valuation of that data as a commodity in driving global digital economies. There are fewer studies that explicitly engage the affective and psychological position of the individual subject in relation to either the identification of self with data presence, or to selfidentification with one's data profile, especially in relation to psychic processes (notable exemptions include Balick 2014b; Krüger \& Johanssen 2014; Singh 2017).

Accessibility of convergent media content, in its various formats, using various platforms and hardware, and from an array of access points, is becoming open to increasingly individual, personalised choices. This condition lies at the very heart of what might be described as a Web 2.0 ethic of connectivity: the notion that media forms are inclusive, participatory, writable from the perspective of an end-user, immediate and, ultimately, democratised through practices of access, sharing and gift economies. At the front-end of these services, it would seem that the extent of that freedom of choice, of paramount importance in a deregulated media ecosystem, signifies an individuated agency that is at once participatory and empowering. However, this position has increasingly become prone to criticism from a number of disciplinary approaches where, even at this front-end of service provision, choice has an illusory dimension (see, for example, Zelenkauskaite 2016). I refer here to the algorithms associated with, for example, massively popular streaming services - specifically, Amazon Prime, Netflix, or YouTube serve as key examples. These services operate within economies of attention and affect to present the consumer with 
front-end suggestions according to not only personal preferences based upon prior consumer choices (a narrowcast-based, 'pull' tendency), but also complexities associated with third-party arrangements for profile and data monetisation, and content provision (a more ostensibly broadcast-based 'push' tendency, partially based on long-tail economic models).

Another case in point here: various algorithms have been developed to increase market potential for YouTube producers - note the use of that term, 'producers', which is a professionalised recuperation of the more interactive, participatory and amateur-ish 'produsage' model often referred to in Web 2.0 scholarship (e.g. Bruns 2008). As if to illustrate the extent of this recuperative turn, for example, DigitasLBi and Outrigger Media developed OpenSlate - social media analytics platform partnered with YouTube and designed to anticipate stars of the future before they break. The results of analysis guide investment choices for corporate players to develop new online talent, and decrease risks of such investments whilst simultaneously increasing the chances of return on investment. Essentially, according to Learmonth (2013), OpenSlate produces predictive, qualitative data of a kind not available from YouTube's own analytics systems. Webster (2014) stated that, at his time of writing, OpenSlate tracked over fifty thousand channels, and twenty-five million individual videos on YouTube, giving scores based upon specific qualitative criteria: audience engagement, frequency of new content added, influence and reach. ${ }^{1}$ For Webster, 'It's possible the talent identified in this way would hit it big without intervention. But using metrics to identify winners can create winners. Unlike the weather, social predictions can change outcomes'; and, unlike in the physical world, predictive 'algorithms powered by big data have the potential to create "self-fulfilling prophecies" in the social world" (2014: 93).

Although writing about a slightly different aspect of the data studies field, one of the more radically-infused book-length critiques of this logic can be found in the aforementioned work of Deborah Lupton - The Quantified Self (2016). To reiterate, Lupton's study explores the forms and practices of self-tracking via various lifestyle applications powered by Web

\footnotetext{
${ }^{1}$ At time of writing, according to OpenSlate's own website, they have the ability to track over 400 million videos on YouTube, illustrating the incremental velocity of scale involved in this sector of advertising. https://www.openslate.com/context/ [Accessed: 21/05/2018].
} 
2.0 connectivity, from a critical sociological perspective. Once again, the mass popularity of these technologies should not be underestimated - this is at the very popular end of popular culture. Such self-tracking practices - the phenomenon of FitBit for example, or any number of applications associated with measurements of body-mass indexing, jogging-route mapping, stepometers, and other wearable technologies or mobile phone applications, all form familiar aspects of everyday media engagement, as well as, one could argue, impression management. The implications of Lupton's work also necessitate that one considers ways in which such practices partake of a broader culture associated with selfimprovement, modification and technologies of well-being or work productivity within relations of power. I have discussed some of the intimate correlations and dialogues between self-image, lifestyle and cultures of self-improvement elsewhere (Singh 2015) so I do not wish to repeat too much on that here, but it is noteworthy that such questions have begun to be addressed from a post-Jungian critique. ${ }^{2}$

Although social media platforms and other Web 2.0 technologies may be identified with affirmative possibilities for expressive freedom, lifestyle, politics (transgression, action or subversion), they equally have tendencies to exacerbate what Laing described as the otherwise 'normal' pathological split between an 'inner' self and a generalized deadness of the embodied 'false-self'. Elsewhere, I have argued that in contemporary media ecosystems, interactions can quickly disaggregate into moral positions of diminished recognition of others as whole persons with unique interests. This false-self attitude is characterised by an objectification of others, an amplification of unreal perceptions, unreal expectations and futile actions, the kinds of which are encountered in, and mobilise online filter bubbles (Singh 2018: n.p.).

This is perhaps expressed best in Laing's vicious circle of falsity; that: 'Love is precluded and dread takes its place', where 'The self by its detachment is precluded from a full experience of realness and aliveness' (1965: 82). Nowhere is this demonstrated to its most complete degree than in the false ideal of body image found in image-driven social media platforms,

\footnotetext{
${ }^{2}$ Indeed, for the range of subjects and positions taken on self-improvement and transformation, see the whole collection within which that specific work sits: Hockley and Fadina (eds.) (2015).
} 
where people are both quick to judge bodies, and quick to call out body-shaming. On the one hand, if we enter into this point of debate, there is a sense of reification of self and other: we are fundamentally disembodied from ourselves in the sense of over-identifying with persona(e) in a false-self system, thus foreclosing on lived, warm relationships based on mutuality. On the other, the body itself becomes a sublime reified object, coded as an idealised unit of exchange, where selfies are currency for the mobilisation of identity in social exchange. Furthermore, such identity is bound up with a notion of data, such that it is reduced to a set of preferences and, ultimately, a quantification of choice (a system of presets and 'preferences'), out of which, contained choices present themselves. In the image-driven emphasis of optic-privileged culture, the evidential value of the selfie (Walker Rettberg 2014; Sturken and Cartwright 2000) as expression of lifestyle should not be underestimated: both in the sense of its representational face-value aspect, and in its monetized data-value aspect. If nothing else, the phenomenon of the selfie demonstrates that we are, in the false-self system of social media, quite literally what we consume; or, we are nothing.

The aspect of Lupton's work that I would like to emphasise in relation to these issues through dialogue with some of the post-Jungian ideas around psychologies of the self I outlined a moment ago, is her emphasis on the ways in which practices of self-tracking are 'spreading from the private realm into diverse social domains, and the implications of the self-tracking phenomenon for the politics of personal data, data practices and data materialisations' (2016: 1). In other words, the intensification of private experience is being drawn into the social. Indeed, we saw a speculative dramatization of this in the chapter's opening example from 'The Entire History of You', where intensely private aspects of the personality (perspective, memory) are being disclosed openly, and relational aspects (sexual congress) are subject to atomised privacy. Additionally, according to some research, this tracking includes intensification of privatised places of work, institutions, and other transactional spaces, where the labouring body is objectified, and the imperative to perform, has become internalised (Moore and Robinson 2015). The vehicle through which this intensification is moving is in the materialisation of data identity. 
This is where the idea of a false-self system starts to make more sense. The quantification (at least, the attempted quantification) of selfhood through standardised systems of profile building, data-mining and so on in everyday online transactions, is a totalised and totalising system of sorts. Underpinned by data identity, social media interactions of various descriptions systematically push towards traffic increase. The most obvious way to achieve this is through pushing what is already conventional and popular, risk-averse, and culturallyimmediate. This totalising, recuperative movement is found in the diversity of cases I have discussed so far in this book. For instance, the impact of multichannel networks often owned by major media multinationals such as Maker Studios (Disney), machinima.com (owned by Time Warner), and the music-oriented content of Vevo (Universal Music Group, Sony Music Entertainment and Warner Music Group). Additionally, the role of microcelebrities, characters and influencers to draw traffic through personality attraction and endorsement (such as Shay Carl Butler, Colleen Ballinger and many others), Instagram and other platform stardom (and in the case of Essena O'Neill, an extreme example of totalising image convention). These visible cases overlay an invisible foundation level of data appropriation, mining, analytics and manipulation operating at the back end of Web 2.0 technologies, driving smart tech interaction, and providing frictionless consumer profiling.

Although on an everyday level as consumers, we may not be aware of, nor necessarily concerned with, the transactional character of these aspects of data-driven identity, these are questions in need of address. As discussed in Chapter Five at length, the experiential, existential human self is not expressive of, nor can it be expressed in, data. Identities of various descriptions might be materialised through data, and this is an interesting notion, but it is misleading to attempt an elision of identity with expressions of selfhood here. An authentic sense of self cannot be sustained through data transactions, nor a sense of self be managed by and through data, for similar reasons. Individuality may be identifiable as and through data-driven systems of identity, but in this kind of 'self system', the notion of self is reduced to the status of ident, brand-presence and lifestyle choice. To refer to previous discussions, in this sense, the 'falsity' of this false-self refers not to fakery or imagined selves, but to a sense of the untrue, and the inauthentic, as in: 'untrue to itself'. Presets and preference settings, vicariously shared through social media updates and ostentatiously pushed through practices such as 'Like-farming', Snapchat 'streaks' and other methods used 
by ordinary users to acquire some aspect of recognitive contact, are in danger of being misrecognised (by systems as well as by users themselves) as expressions of the whole person. As we have seen in the various discussions on recognition offered over the course of this book, this is a fundamental ethical and existential error.

If further support for this argument is needed, we might appeal to Hegelian intersubjectivity and Hegel's well-known account of the struggle for recognition in Phenomenology of Spirit (1994 [1807]) to tease out what is necessarily 'false' about this particular kind of 'self system'. This argument is relevant as an influential precursor to relational psychology's intersubjective interaction between more than one psyche as meaningful interaction. Following Aristotle's notion that one's relationship to others always runs parallel to one's relationship with oneself, Hegel's idea of self-consciousness is as a self-consciousness existing only as something recognised. The need for others outweighs every other need or desire, and this is a crucial precondition in any society that pertains to conviviality as a 'living together' or 'living well' ethical value. Hegel writes that 'Self-Consciousness must orient itself toward superseding the other independent being, in order to become certain of itself thereby, as the being that it is; it must concomitantly be oriented toward superseding itself (since this "other" is itself)' (1994: 51, §180 [emphasis in original]). In other words, selfconsciousness must negotiate with an other (self-)consciousness, recognising itself in the other in order to become certain of itself, so that eventually, 'The consciousnesses recognize themselves as mutually recognizing each other' (1994: 53, §184 [emphasis in original]). However, false-self systems seek, through this process, to define selfconsciousness as individuated, and this mutuality is subject to various intermediaries of the kinds just described. This intermediation is a problem for a consciousness that desires to be recognised as itself, above all else. As (Hegel translator) Kainz notes (1994: 50, note 5) in his annotated edition of Phenomenology of Spirit:

[...] a human being's existence as a self-consciousness is in a very real sense dependent on recognition by alter egos; so that if [...] someone were to exist without any recognition at all (not even of a negative sort), this person would not exist as a self-consciousness. 
An argument could be made here that, in a media ecosystem defined through social interactions that are mediated through data materialisation, the moment and sustaining of recognition in order to maintain a sense of life and security overrides other principles (and this could include moral ones). Such an argument also recognises that freedom (to act, speak, assemble - in general, but also specifically, in social media interactions and Web 2.0 activities) is a commodity in the exchange of recognition. It permits, in a synoptic sense, the licence to say and act in ways that surpass moral value and social obligation. This is because, in a recognition economy driven through data materialisation, one's only obligation would be to recognise another as another would recognise you - however negative or alienated.

In human interactions, healthy personas are characterised by both robustness and strong differentiation. However, social media communications have an in-built tendency for communication shorthand, and this tendency systematically favours the personally convenient, and the immediacy of identification with persona as a quick-response, 'easy version' of the self. On this basis, assumed versions of oneself and others have a tendency to become estranged; and relations become objectified. Self, in this sense, is systemic and simulated, rather than expressed in any strong sense by the user; and the routines that characterise the practices of 'quantified self' movement (Lupton 2016) are the governing principle at the data level in all social media platforms (Singh 2017).

To take trolling as an example of this sort of objectification that lies upon a more visible level to the user (i.e. comments threads), although an act ostensibly subject to popular criticism trolling also becomes, controversially, something of an exonerated act. This is because it ostensibly fulfils the logic underpinning obligations of recognition in the most superficial, non-mutual sense: I recognise you, albeit negatively, because I either respond to something you have posted online, or, I deliberately provoke you into a false debate on a controversial subject. It also expresses a vulgar definition of freedoms of speech (advocates of which, have been popularly described in online contexts as 'speechers') without fully acknowledging the responsibilities that necessarily associate themselves with those freedom rights: you recognise me because, albeit negatively, I have expressed a right to freedom of speech. In such unfortunate, somewhat solipsistic circumstances, in the practice 
of trolling the consciousnesses do not recognise themselves as mutually recognising others with independent and unique interests.

The problem here rests on the notion that recognition is not only affirmative in the positive sense of life affirmation but is also subject to, and circumscribed by, conditions through which that recognition takes place (a commodified and commodifying environment). In this case, a data materialisation is built within various instances of capital (economic, social, cultural, and - perhaps most immediate and potent given the mediated environment of social interactions in Web 2.0 contexts - symbolic). Additionally, the limits of recognition are also shaped through the predominant value system: a value system that takes freedom of speech to its limits and beyond, whilst ignoring the parameters of responsibility and justice associated with those rights in moral and legal frameworks is a value system that, however misplaced, exonerates trolling and other forms of cyberbullying. Essentially, what we are dealing with therefore, is a variation on Hegel's 'unhappy consciousness', described by Robert Sinnerbrink as an alienated form of subjectivity: 'As Hegel contends, however, alienation can be overcome only through the rational comprehension of our historical condition and through the social achievement of mutual recognition' (2007: 55 [my emphasis]). So, whereas it may first appear that dialectical movement of recognition is corrupted through the conditions of commodity-identity and data materialisation prevalent in social media interaction, that effectively fuels corrosive practices such as trolling, the principle of mutuality once more provides a solution. This time, mutuality augments the self-psychology of Kohut and Benjamin, and the philosophical anthropology of Honneth, with an appeal to Hegelian phenomenology, and to his philosophy of right.

This leads us to another aspect of Laing's work that fully appreciates the necessity to engage productive and positive aspects of interpersonal interaction. In addition to the vicious circle of the false-self, Laing describes mutual recognition between persons as a benign circle, a 'mutual enrichment of self and other'. He writes that 'The reality of the world and of the self are mutually potentiated by the direct relationship between self and other' (1965: 82). For Laing, this mutuality forms the intersubjective basis of an existential equilibrium. He furthers this observation by emphasising the fundamental expression of mind, body and world as a creative expression of consciousness, in a relationship with other 
consciousnesses. However, without a principle of mutuality guiding the full recognition of others as whole persons with autonomous interests, this expression is impossible. He states (1965: 84) that without such mutuality,

What one might call a creative relationship with the other, in which there is a mutual enrichment of the self and the other (benign circle) is impossible, and an interaction is substituted which may seem to operate efficiently and smoothly for a while but which has no 'life' in it (sterile relationship). There is a quasi-it-it interaction instead of an I-thou relationship. This interaction is a dead process. (1965: 82 [emphasis in original]).

To avoid dead, 'quasi', inauthentic interactions, and to reinvigorate the I-thou status of fully recognitive being, Laing's creative relationship necessitates a leap of faith to retain a warm, lived sense of mutuality. I argue that it involves risk in revealing enough of oneself to another to facilitate the openness that trust requires to establish mature relationships. It also requires a full recognition that the contemporary communications media ecosystem does not necessarily have the capacity to facilitate such openness, because of the representational regime and data materialisation of online identity as if it were the whole thing (a self). To borrow once more from Aaron Balick, 'Pathology develops only when the individual identifies with their persona at the expense of other attributes of their personality: when they believe the persona to be 'the whole thing' (2014b: 16). The 'pathology' at work here, is the inauthentic false-self.

\section{Social Media and the Complex of Authenticity}

Following Joseph Henderson's pioneering typology of social, aesthetic, philosophical and spiritual dynamics, the psychiatrist and Jungian analyst Thomas Singer $(2000,2004)$, along with his colleague Samuel L. Kimbles (2000) developed the concept of the 'cultural complex'. This is a term which derives specifically from the notion of 'complex' found in the analytical psychology of Jung and post-Jungian thought. In this school, complexes operate at the personal level, and are made up of the building blocks of the psyche, often experienced as partial personalities or distinct psychological entities. Ultimately, these experiences 
derive from the tensions and interplay between archetypal, unconscious materials in the psyche, and the necessity to deal with the outside world. The term 'complex' is often used in depth traditions to describe the constellation of affects, images, emotionally-charged associations and collective psychic material.

The 'cultural complex' is a development of the original term in post-Jungian thought and psychotherapy. It came about through a necessity to acknowledge the role of interaction between intrapsychic processes of individuals and those of others; and, also between the psychic world and the world of the social in general. The cultural complex describes powerful moods and behaviours characterised by repetition, lived out at a cultural level as part of a zeitgeist of attitudes, behaviours and worldviews. In time, these tend to become internalised by individuals through various identification processes, and accepted as certain ways of thinking about and doing things (although crucially, remaining not necessarily the best way to think and do). Singer writes that, 'For Jungians, Henderson's work opened the door to the vast realm of human experience that inhabits the psychical space between our most personal and our most archetypal level of being in the world' (2004: 19). He goes on to suggest that, 'like personal complexes, cultural complexes provide a simplistic certainty about the group's place in the world in the face of otherwise conflicting and ambiguous uncertainties' (2004: 21). It is a process whereby one's own sense of self mingles with larger notions of identity in a collective sense, and then further: when one's own cultural complexes mingle with other cultural complexes in reciprocal amplification. As Singer (2004: 32) writes:

We hold up strange mirrors to ourselves and to one another when we start to explore cultural complexes as part of our personal and historical development. Our cultural complexes get all mixed up not only with our personal history and complexes but with other cultural complexes as well.

There is, arguably, a more materialist outlook to the notion of cultural complex when compared to the classical Jungian notion of personal complex: it tends to emphasise the social, and may be regarded as fundamentally historical in its character, as it reflects diachronic shifts in modes of worldview, shaping norms and values over time. However, this 
is a sense of personal or shared history that is present, that is unfolding as one interacts with others and with the world. It is almost as if an aesthetic of being operates through this experience and gives one a sense of being present, and alive in the moment. It is therefore a powerfully seductive aspect of authenticity and carries with it a sense of both identity and recognition.

I would argue that the notion of authenticity, although certainly mythic in character (certainly in the kinds of aesthetic experiences just described), and problematic in its relationship with essentialist truth claims and so on, relates closely to the notion of cultural complex. This is particularly the case when the struggle for authenticity, for a feeling that is true to oneself, is amplified through social media interactions. Indeed, one might tentatively argue that authenticity is itself an instance of complex. I have noted elsewhere, for example, that persona tends to amplify the individual, and likewise, the interaction of cultural complexes will amplify and extend conventions of worldview, and of social interaction. So, persona on an interpersonal level (the psychological operations that reveal and conceal to keep the personality intact), and cultural complexes of authenticity at a wider level (that address the social necessity to express and remain 'true' to oneself, in the individualist sense) together, distort the view of the true self for others. However, as I have mentioned elsewhere (Singh 2017) it is never fully a false-self that is presented. The term 'false' is not intended to signify 'inauthentic'. As already noted in a number of contexts during the course of this book, inauthenticity as a concept ought to be reserved for cases where individuals and others identify with the false-self as if that is all there were. This appears to be a characteristic of zeitgeist, and reflects the complexity (and arguably, the complex) of the struggle for authenticity.

In order to complete this argument, I turn once again to the thought of Charles Taylor. His work on the intensification of privatised experiences, and the inflated cultural value of what he terms 'self-realisation' (closely related to the concepts and practices of individualism, self-actualisation, and 'lifestyle' individuation), is of particular interest here. He writes that the contemporary culture of authenticity, which characterises modern life, 'encourages a purely personal understanding of self-fulfilment, thus making the various associations and communities in which the person enters purely instrumental in their significance. At the 
broader social level, this is antithetical to any strong commitment to a community' (1991: 43). This increasingly intensified, private experience intimately fosters a transactional approach to relationships, where one's relationships are viewed as being subservient to personal fulfilment. For Taylor, as it stands, the predominant and impoverished principle of individualism does not offer a view on how individuals should live with others. It is a curious echo of the convivialist ethos, coupled with echoes of Hegel's struggle for recognition, as well as the psychosocial principle of mutuality that I have mapped out in this book. Taylor's acknowledgement of the problem speaks to an unfolding and unfoldedness of intersubjective (social) being that seems to be all but absent in the transactional and data-driven versions of selfhood promoted through social media interactions. He writes (1991:47-48) that

My discovering my identity doesn't mean that I work it out in isolation but that I negotiate it through dialogue, partly overt, partly internalized, with others. That is why the development of an ideal of inwardly generated identity gives a new and crucial importance to recognition. My own identity crucially depends on my dialogical relations with others.

That he had already identified such problems in the 'culture of authenticity' many years before the advent of social media, and indeed predating the Web by some several years, the amplified effects of such impoverished recognitive forms within contemporary always-on contexts gives cause for concern. But this also provides an opportunity to engage with some of the solutions laid down in a number of traditions of thought and praxis discussed at length during the course of the current study. Taylor is optimistic. He argues that it is difficult to believe that people are so locked into social and material conditions such as the atomist, instrumental, transactional relations that seem to characterise modern life, that they are not able to make positive change, and embrace a more authentic approach to relationships, to community, and to a historical sense of self and place. He states (1991: 73) that 
[...] while everyone must recognize how powerfully we are conditioned by our industrial technological civilization, those views that portray us as totally locked in and unable to change our behaviour short of smashing the whole 'system' have always seemed to me wildly exaggerated.

As any reader who has read thus far would be able to discern, I have some sympathy with this view. Indeed, so does Jaron Lanier in a couple of distinct ways: expressions of his vehement anti-Marxism (2006), aside (which in some ways nonetheless align with Taylor's call to retain the current social apparatus, but transform its content), the strong individualist stance that Lanier expresses in many of his published projects $(2010,2013)$ has much in common with Taylor's view. Furthermore, the language that Taylor chooses here is significant for Lanier. 'Lock-in' is, of course, a technical term widely used in the field of human-machine interactions. It is used by Lanier in that sense, but he also uses it to extrapolate the trajectory of human-machine interactions, to attempt to figure out how and why human-human relationships, and the interactions individuals have within wider communities, correlate with locked-in technological and social practices. Again, in an echo of Lanier's critique of degraded social interactions on SNS platforms, discussed in some detail in previous chapters, Taylor suggests a methodology to address the 'debased' practices of misrecognition. He writes (1991: 72) that we need to

[...] undertake a work of retrieval, that we identify and articulate the higher ideal behind the more or less debased practices, and then criticize these practices from the standpoint of their own motivating ideal. In other words, instead of dismissing this culture altogether, or just endorsing it as it is, we ought to attempt to raise its practice by making more palpable to its participants what the ethic they subscribe to really involves.

This necessitates a meaningful involvement in taking up an ethical position, and again, in making a leap of faith. The future may appear to offer only ever-increasing levels of inwardlooking, privatised concerns. It may be the case that a variety of factors, including increased precarity, enforced mobility, and jobs and situations that demand we act or behave more 
transactionally towards one another, all characterise contemporary lifestyles, particularly in the so-called developed world. This is a depressing thought, and if taken in the rather mean spirit of much popular commentary where, particularly young people, are inwardly and narcissistically concerned with their own intensely privatised concerns, then it is true for Taylor that we are indeed running out of alternatives to reverse such trends. However, to affirm Taylor's optimism, he also contends (1991: 76-77) that:

This perspective is different if you see these developments in the light of the ethic of authenticity. For then they don't just represent a shift in value that is unproblematic for the people concerned. Rather, you see the new, self-centred practices as the site of an ineradicable tension. The tension comes from the sense of an ideal that is not being fully met in reality. And this tension can turn into a struggle, where people try to articulate the shortfall of practice, and criticize it.

Struggle means that things can go either way, which for Taylor at least means that there is good news and bad news. But this struggle brings with it the potential to push forwards, towards the ideal, and that decline and triviality are not inevitable. And again, this suggests that same leap of faith. But this leap also requires a sense of the social imaginary - the place where Laing's 'creative relationship' is allowed to flourish and express itself to the fullest degree, and where a sense of conviviality can gain social aspects that reinforce the psychological. I am here minded of Sartre's work in The Psychology of The Imagination (2001). In his book, Sartre writes (2001: 169 [emphasis in original]) that

To prefer the imaginary is not only to prefer a richness, a beauty, an imaginary luxury to the existing mediocrity, in spite of their unreal nature. It is also to adopt 'imaginary' feelings and actions for the sake of their imaginary nature. It is not only this or that image that is chosen, but the imaginary state with everything it implies; it is not only an escape from the content of the real (poverty, frustrated love, failure of one's enterprise, etc.), but from the form of the real itself $[\ldots]$ the very way our feelings have of developing themselves. 
This is an expression of authenticity in its positive, truest sense. It is an opt-in, positive solution to what is often seen as a negative problem, demanding subtractive or censorious solutions. In the context of Web 2.0, these taken the familiar discursive form of a number of everyday issues - moral panics about children with technology, for example; the surveillance anxieties of modern state and corporate data practices; or the accompanying paternalist censorship that forms around National Webs and so-called safe spaces. Seeing the imaginary in this creative way transforms our understanding of social being from one dominated by a transactional, neoliberal economism that dominates cultural life today, to one that seems, through the imaginative world of recognition, more to do with the expression of interior lives, and the potentialities of convivial, lived mutuality. That transactional culture, to which I have alluded several times throughout this book, underpins the majority of social interactions via Web 2.0 technologies through data materialisation and the fixity of image-driven social pressures. Holding up Sartre's reading of the imaginary and Laing's notion of the creative relationship with the other, one can see another path to tread.

Our intuition may tell us that we are warm, living, social, emotional, sensible, animated, thinking things; that we are, in fact, human beings, (mostly) being human. Whereas the Black Mirror instance at the beginning of this chapter may well be an extrapolation of existing affordances between technology, the sexual and the social, nonetheless, one can see the ways in which technological intermediaries compel us towards reified relations, amplifying an already delicate state in the intrapsychic balance between self and other. Indeed, a more materialist argument might suggest that our human relationships in general are thus in danger of becoming relations between things - the 'being' part of 'human being' melting into air as the objectification mechanisms of commodity-identity and reification become more prevalent. Indeed, at the height of modernity, these mechanisms were identifiable, well-known even. In History and Class Consciousness, for example, Georg Lukács, (1990: 83) wrote that

The essence of commodity-structure has often been pointed out. Its basis is that a relation between people takes on the character of a thing and thus acquires a 
'phantom objectivity', an autonomy that seems so strictly rational and all-embracing as to conceal every trace of its fundamental nature: the relation between people.

In this phantasmal, transactional rationality, Lukács identified commodity as a sort of structuring principle of everyday life, defining our sense of individuality, and well as setting the terms for social, institutional and political relations. To put it another way, our warm, lived psychology is disassembled through a reduction to 'thingness'. However, because we are not really things in the sense of inanimate, inorganic or cold things our intuition should push us towards finding a solution to accommodate the desire for recognition as whole persons with autonomous and unique interests. One way of dealing with this, tarrying with materialist terminology of commodity, is to develop the notion that commodity is a structuring principle by reimagining commodity as a recognitive principle of mutuality. Whether we can get to this point in our dealings with the legacy of Web 2.0 technologies, practices and principles is another question, and in my concluding remarks, I tentatively offer some explorations. 